\title{
The microcirculation
}

\section{NE Hendricks iD}

Department of Anaesthesia, Charlotte Maxeke Johannesburg Academic Hospital, University of the Witwatersrand, South Africa Corresponding author, email: nicole.hendricksmd@yahoo.com

Keywords: microcirculation, endothelial glycocalyx

\section{Introduction}

The microcirculation is responsible for the transfer of oxygen from the red blood cells in the capillaries to the cells to meet cellular energy requirements, support functional activity and remove carbon dioxide and waste. The microcirculation also assists in the regulation of vascular tone, solute exchange, the production of hormones, the inflammatory response and haemostasis. The identification of the endothelial glycocalyx and the assessment of the effects of disease, drugs and fluids on the microcirculation is the subject of ongoing research.

\section{Structure and physiology}

The microcirculation includes arterioles, metarterioles, capillaries and venules. ${ }^{2}$ These vessels have a diameter less than $100 \mu \mathrm{m}$. There are small (lower than $20 \mu \mathrm{m})$, medium $(20-50 \mu \mathrm{m})$, and large $(50-100 \mu \mathrm{m})$ vessels.

The microcirculation surface consists of endothelial cells that interact with a collagen rich basement membrane. The glycocalyx $x^{3}$ and the blood are in dynamic equilibrium. Cells contain fenestrations and pores and are held together by molecules, e.g. cadherins. They also contain current-carrying gap junctions. This allows upstream electrical communication between endothelial cells. ${ }^{4}$ The morphology of the endothelium varies between different organs.

The glycocalyx is a semipermeable membrane. ${ }^{3}$ It is a $0.2-$ $0.5 \mu \mathrm{m}$ gel-like negatively charged layer synthesised by endothelial cells. It has $\mathbf{3}$ major components - proteoglycans,

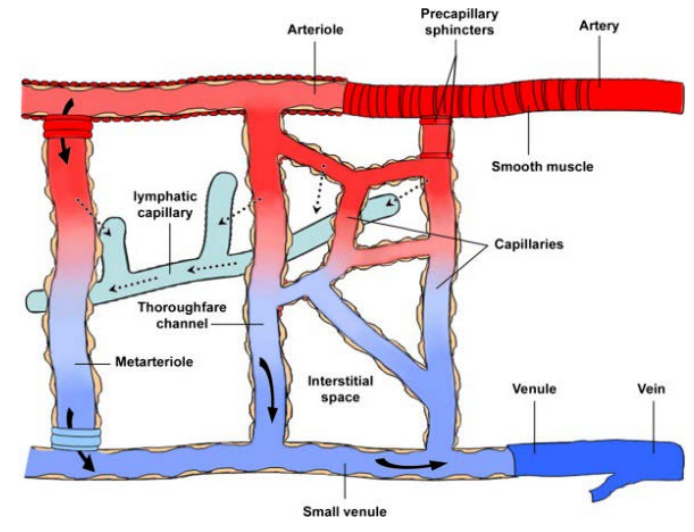

Figure 1: Schematic representation of microcirculation components ${ }^{1}$ glycosaminoglycans, and plasma proteins. It also contains substances such as antithrombin and superoxide dismutase. Glycoproteins, such as selectins, integrins and immunoglobulins, anchor this glycocalyx layer.,4 Many of the functions of the microcirculation come from the glycocalyx. ${ }^{4}$ It allows for endothelial-leucocyte interaction, cell-cell adhesion and mechanical transduction as well as integration of leucocytes and platelets respectively. The net negative electrostatic charge also results in platelets being repelled as well as anticoagulation. The glycocalyx permits molecules with a molecular weight of < $10000 \mathrm{Da}$. Water and electrolytes are able to pass through the glycocalyx but plasma proteins like albumin require specialised pores.

\section{Regulation of blood flow and oxygen transport}

The endothelial cells together with smooth muscle cells regulate the microvascular blood flow predominantly by regulation of vascular tone. ${ }^{5}$ This is by three main mechanisms of autoregulation: myogenic, metabolic and neurohumoral control.

Myogenic - stretch due to intrinsic contractile response. It is mediated via deactivation of K-ATP channels that regulate membrane potential, and then propagation of the signal.

Metabolic - build-up of vasodilator substances, e.g. $\mathrm{CO}_{2}$, lactate, adenosine, $\mathrm{H}^{+}$, decreased $\mathrm{pO}_{2}$ via hypoxia inducible factor 1 alpha.

Neurohormonal - autocrine and paracrine interactions from 5-hydroxytryptamine, bradykinin, cytokines, GFs.

The vascular bed of skeletal muscle has rich adrenergic innervation and therefore has a vasoconstrictor response to neural stimulation, primarily through the resistance vessels. Precapillary sphincters also constrict in response to sympathetic stimulation, but are sensitive to local $\mathrm{pO}_{2}, \mathrm{pCO}_{2}, \mathrm{pH}$, potassium, magnesium, osmolarity and adenosine.

Regulation of blood flow and oxygen delivery in response to increased oxygen demand and reduced oxygen delivery are accomplished by three functionally distinct portions of the microcirculation: 


\section{Resistance vessels}

\section{Exchange vessels}

\section{Capacitance vessels}

The resistance vessels are the muscular arterioles that control regional blood flow. The alteration in the smooth muscle tone is responsible for active constriction and dilation in arterioles.

The capillaries are responsible for the exchange function in the circulation. Small discontinuous bands of vascular smooth muscle, the precapillary sphincters, are located at the arterial end of many capillaries and are responsible for the control of blood flow within the capillaries. They are lined by a single layer of endothelial cells and are responsible for the exchange of oxygen and nutrients between the intravascular and adjacent cells. In resting conditions, only $20-30 \%$ of the capillaries are functional and are actively participating in tissue perfusion. With tissue hypoxia, capillary recruitment occurs rapidly due to the opening of precapillary sphincters. This allows for the maintenance of a dynamic environment for exchange.

The venules act as the capacitance vessels, which collect blood from the capillary network and function as a reservoir for blood in the circulation. Their distensibility and high capacitance allows for storage and mobilisation of large amounts of blood. This blood can be centralised by activation of the physiological volume regulatory mechanisms (sympathetic nervous tone, vasopressin, natriuretic hormones, vascular permeability, and lymphatic return) and this is where the body initially deposits $80-85 \%$ of any infused volume.

Vasoactive hormones (e.g. renin, vasopressin, prostaglandins and kinins) also play a role in microvascular control. The endothelial cells themselves produce vasoactive molecules responsible for regulating arteriolar contraction and help with blood pressure control.

Endothelial vasodilating substances - Nitric oxide (NO) and prostacyclin. NO produced with nitric oxide synthase (NOS), includes endothelial NOS (eNOS). ${ }^{6}$ It activates smooth muscle via cGMP pathway and protein kinase G. It is stimulated by ADP, bradykinin, muscarinic agonists, shear stress (including exercise), and is also involved in vascular remodelling, angiogenesis and atherosclerosis. eNOS and inducible NOS (iNOS) imbalance during sepsis is an important mediator of microcirculatory vasodilation, where areas without NO are underperfused.

Endothelial vasoconstricting substances - Thromboxane $A 2$, endothelin and platelet activating factor (PAF). Endothelin -1 secreted locally and released near to damaged endothelium. Regulation of secretion is via angiotensin II, catecholamines, shear stress and hypoxia with stimulation of gene transcription, and NO and prostacyclin which inhibit gene transcription. Prostacyclin and thromboxane A2 balance is important for localised platelet aggregation and clot formation while preventing excessive clot extension and maintaining blood flow around the clot.
The mechanical autoregulation of blood flow countering changes in blood pressure allows blood flow to chosen organs (brain, heart, kidney, liver, carotid bodies) to remain constant over a wide pressure range, provided there is no change in organ function.

\section{Haemostasis and coagulation}

Endothelium participates in platelet activation, the coagulation cascade and fibrinolysis. Vasodilators such as prostacyclin, nitric oxide and ADPases can be liberated. Mediators like shear stress, hypoxia and cytokines induce expression of tissue factor and the tissue factor pathway. With vascular injury, vasoconstriction concentrates repair, and injured endothelium further exposes von Willebrand factor (vWF) and collagen in order for a platelet plug to develop.

The proteoglycans making up the glycocalyx consist of a core protein to which GAG chains are linked. Proteoglycans can bind to any of the five GAG chains:

1. Heparin sulphate - potentiate ATIII and heparin cofactor II

2. Chondroitin sulphate

3. Dermatan sulphate - potentiate ATIII and heparin cofactor II

4. Keratin sulphate

5. Hyaluronic acid

Heparin sulphate makes up $50-90 \%$ of the total amount of proteoglycans present and is a co-factor for antithrombin III, amplifying its antithrombotic properties.

Receptors and enzymes affect cell signalling in the glycocalyx. The healthy glycocalyx is protective as it binds ATIII, TFPI and thrombomodulin, thus making it thromboresistant. Its negative charge also repels platelets.

- Heparin sulphate and dermatan sulphate

- Tissue factor pathway inhibitor - binds Xa and inhibits TF-aVII complex

- Thrombomodulin - binds protein C and increases anticoagulant activity with protein $\mathrm{S}$

- Accelerated activation of protein $C$, via endothelial protein $C$ receptor. This then inhibits factor V and VII

- Tissue plasminogen activator -catalyses the conversion of plasminogen into plasmin and fibrinolysis

\section{Regulation of solute exchange}

The capillaries and post capillary venules are the sites where fluid and solute exchange with the interstitium occurs. Transfer of substances through the capillaries can occur directly through the endothelial cell or via intercellular pores in the capillary membrane. Capillaries have varying permeability depending on the organ they perfuse. They are described by the differences in their endothelial linings.

- Continuous/non-fenestrated (CNS, muscle, connective tissue, lung) - paracellular transport through interendothelial clefts; 
the endothelium is linked together by dynamic junctions adherens and tight junctions.

- Discontinuous/sinusoidal.

- Fenestrated (renal glomeruli - (discontinuous glycocalyx), choroid plexus, endocrine organs, GIT) - have additional transcellular holes for increased molecular exchange between intra and extracellular compartments.

The two principle means of accomplishing exchange of substances between tissue and blood are diffusion and filtration.

Simple diffusion is important for the respiratory gases and water flux across the blood-brain barrier via aquaporins. Paracellular filtration allows generally low but permanent fluid flux outwards into the interstitial space.

\section{Starling's forces}

Described for peripheral microvascular beds with a continuous endothelium (not discontinuous).

\section{What we knew}

It was incorrectly assumed that fluid is filtered into the interstitium via the capillary, and then reabsorbed as it approaches the venules.

- Starling's principle stated that:

。 $\mathbf{J v}=\mathbf{K f}[(\mathbf{P c}-\mathbf{P i})-\sigma(\Pi c-\Pi i)]$

- Jv = outward filtration force for a given area, $\mathrm{Kf}=$ membrane hydraulic conductivity, $\mathrm{Pc}=$ luminal hydrostatic pressure, $\mathrm{Pi}=$ interstitial hydrostatic pressure, $\sigma=$ macromolecule reflection coefficient of the membrane, $\Pi c=$ luminal osmotic pressure and $\Pi \mathbf{i}=$ interstitial osmotic pressure.

The degree of leak is quantified by the reflectance coefficient (o) which ranges from 0 to 1 ; where 1 is $100 \%$ reflection with no leak for that solute. This reflectance coefficient differs in different tissues, e.g. blood-brain barrier.

\section{What we now know}

The glycocalyx provides the first and most important resistance to the passage of fluids and solutes. Limiting fluid filtration is related to the oncotic pressure difference that is built up between the intravascular space and a small protein-free zone beneath the protein-loaded endothelial glycocalyx. This proteinfree zone has changed our understanding of fluid flux from the traditional Starling equation..$^{7-9}$

An intact endothelial surface layer is needed. It is important to consider preventing intravascular hypervolaemia, limiting inflammation and limiting ischaemia. Endotoxemia damages the endothelial cell, damaging the glycocalyx integrity and potentially resulting in insufficient tissue perfusion.

$\pi \mathrm{i}=\pi \mathrm{g}=$ sub-glycocalyx colloid oncotic pressure, NOT interstitial fluid colloid oncotic pressure
Major modifications to the Starling principle with a focus on the endothelial glycocalyx. ${ }^{7-9}$

\section{- No absorption in the steady state}

Absorption through venous capillaries and venules does not occur, fluid is removed from the interstitium via the lymphatic system. The interstitial protein concentration has minimal effect on the direction of fluid movement. ${ }^{10}$

\section{- The sub-glycocalyx space}

Interstitial colloid oncotic pressure is less important. The glycocalyx is a molecular filter and selectively alters areas of oncotic pressure. It also has a high reflectance coefficient and reflects large molecules, e.g. albumin.

Predicted and measured filtration rates are resolved by replacing $\Pi i$ with the osmotic pressure in a small protein-free zone between the endothelial glycocalyx and the endothelial cells.

$$
\begin{aligned}
& \text { - Starling's principle now: } \\
& \mathbf{J v}=\mathbf{K f}[(\mathbf{P c}-\mathbf{P i})-\boldsymbol{\sigma}(\boldsymbol{\Pi} \mathbf{c}-\mathbf{\Pi g})]
\end{aligned}
$$

Where $\Pi g$ is the sub-glycocalyx osmotic pressure. As the osmotic pressure counteracting $\Pi \mathrm{c}$ is $\Pi \mathrm{g}$, and not $\Pi \mathrm{i}$, changes in $\Pi \mathrm{i}$ will have little impact on the filtration rate, as has been observed. $\Pi \mathrm{g}$ is almost negligible in comparison to $\Pi \mathrm{c}$, so the osmotic pressure gradient approaches $\Pi c$.

- The endothelial glycocalyx is a determinant of hydraulic conductivity.

- The modified Starling model is non-linear at low filtration rates.

How important is this for anaesthesia?

\section{Pathology}

Endothelium dysfunction is seen in states of shock. Changes in the microcirculation are due to inflammation, activation of coagulation and the complement system and damage to the capillary endothelium by intravenous fluids, sepsis, surgery and metabolic states such as hyperglycaemia. This results in subendothelial oedema.

\section{Septic shock}

Imbalances between vasoactive substances cause vasomotor instability and regional hypoperfusion. There is increased permeability which may lead to tissue oedema, reduction in erythrocyte deformability and release of NO contributing to microcirculatory dysfunction. Changes are both quantitative (reduced vessel density) and qualitative (blood flow - slowed, intermittent, or even stopped). The increase in the distance between capillaries and cells makes hypoxia appear quickly. Adrenergic receptors are less responsive.

\section{Cardiogenic shock}

Reductions in the diameter of arterioles and the functional capillary density. In patients with heart failure, intravenous 
infusion of nitroglycerin may increase functional capillary density, despite a reduction in cardiac filling pressures. Microcirculation may be independent from microcirculatory variables.

\section{Monitoring the microcirculation}

Monitoring the arterial serum lactate, mean arterial pressure, central venous pressure, cardiac output, mixed venous saturation and central venous oxygen saturation $\left(\mathrm{ScvO}_{2}\right)$ may be used to assess critically ill patients. ${ }^{11}$ These are systemic or global perfusion parameters and they do not reflect organ or regional blood flow. There may be a dissociation between these parameters and the state of microcirculation in this group of patients. Fluid resuscitation may increase convection (flow) but at the expense of diffusion of oxygen due to the diffusion distance. ${ }^{11}$ The persistence of microcirculatory changes is associated with higher mortality. This microcirculatory shock requires an assessment beyond that of simply monitoring systemic haemodynamic and oxygen transport parameters.

Additional assessment of the microcirculation may include peripheral perfusion index, temperature gradient, sublingual and subcutaneous carbon dioxide levels, laser doppler flowmetry, tissue oxygen assessment electrodes, videomicroscopy (orthogonal polarisation spectral imaging, sidestream dark field imaging or incident dark field illumination) and near-infrared spectroscopy.

Peripheral perfusion rate: pulse oximetry is used and is able to distinguish between the pulsatile (blood) and the nonpulsatile (other tissue) components and between haemoglobin and oxygenated haemoglobin. Calculation of the index is independent of the oxygen saturation value. Peripheral perfusion index values of less than or equal to 1.4 are associated with tissue hypoperfusion.

Capillary refill time: a time of 5 seconds is the upper limit. It may only be a good predictor of dehydration, reduced systolic volume and increased serum lactate in children.

The monitoring and optimisation of tissue perfusion by direct viewing and microcirculation assessment may be a goal to achieve in the haemodynamic resuscitation of critically ill patients.

\section{Therapeutics and the microcirculation}

Statins increase basal NO production by eNOS rather than iNOS to restore the balance. NO donors, such as nitroglycerin, may improve cardiogenic shock, but not septic shock.
An aPC administration during experimental endotoxaemia improves intestinal microcirculation. However, aPC was removed from use after the PROWESS-Shock trial because it failed to reduce mortality, compared to placebo in septic shock.

Vasopressin (terlipressin $=$ a vasopressin analogue, relatively selective for $\mathrm{V} 1$ receptors) as a vasopressor in septic shock unresponsive to catecholamines may benefit some areas of the microcirculation and cause harm in others.

Levosimendan has been shown to be better than dobutamine in stabilised septic shock patients.

Red cell transfusion is a means to increase NO.

\section{Conflict of interest}

The author declares no conflict of interest.

\section{Funding source}

None.

\section{ORCID}

NE Hendricks (iD https://orcid.org/0000-0003-3126-0246

\section{References}

1. Scioli MG, Bielli A, Arcuri G, Ferlosio A, Orlandi A. Ageing and microvasculature. Vasc Cell. 2014;6:19. https://doi.org/10.1186/2045-824X-6-19

2. Lenasi H. Introductory Chapter: Microcirculation in Health and Disease, Microcirculation Revisited - From Molecules to Clinical Practice: IntechOpen 2016. https://doi.org/10.5772/64680

3. Pries AR, Secomb TW, Gaehtgens P. The endothelial surface layer. Pflügers Archiv. 2000;440(5):653-66. https://doi.org/10.1007/s004240000307

4. Guven G, Hilty MP, Ince C. Microcirculation: Physiology, Pathophysiology, and Clinical Application. Blood Purificat. 2020;49(1-2):143-50. https://doi. org/10.1159/000503775

5. Jacob M, Chappell D, Becker BF. Regulation of blood flow and volume exchange across the microcirculation. Crit Care. 2016;20(1):319. https://doi.org/10.1186/ s13054-016-1485-0

6. Gutterman DD, Chabowski DS, Kadlec AO, et al. The Human Microcirculation - Regulation of Flow and Beyond. Circ Res. 2016;118(1):157-72. https://doi. org/10.1161/CIRCRESAHA.115.305364

7. Woodcock TE, Woodcock TM. Revised Starling equation and the glycocalyx model of transvascular fluid exchange: an improved paradigm for prescribing intravenous fluid therapy. Br J Anaesth. 2012;108(3):384-94. https://doi. org/10.1093/bja/aer515

8. Woodcock T. Plasma volume, tissue oedema, and the steady-state Starling principle. BJA Educ. 2017;17(2):74-8. https://doi.org/10.1093/bjaed/mkw035

9. Levick JR, Michel CM. Microvascular fluid exchange and the revised Starling principle. Cardiovasc Res. 2010;87:198-210. https://doi.org/10.1093/cvr/cvq062

10. Milford EM, Reade MC. Resuscitation Fluid Choices to Preserve the Endothelial Glycocalyx. Crit Care. 2019;23(1):77. https://doi.org/10.1186/s13054-019-2369-x

11. Tafner P, Chen FK, Rabello RF, Corrêa TD, Chaves RCF, Serpa AN. Recent advances in bedside microcirculation assessment in critically ill patients. Rev Bras Ter Intensiva. 2017;29(2):238-47. https://doi.org/10.5935/0103-507X.20170033 